\title{
Survey of veterinarians in the Netherlands on antimicrobial use for surgical prophylaxis in dairy practice
}

\author{
R. Jorritsma, ${ }^{1 *}$ A. Van der Heide, ${ }^{1}$ and I. M. Van Geijlswijk ${ }^{2}$ \\ ${ }^{1}$ Faculty of Veterinary Medicine, Department of Population Health Sciences, Division Farm Animal Health, Utrecht University, 3584 CL, Utrecht, \\ the Netherlands \\ ${ }^{2}$ Faculty of Veterinary Medicine, Department of Population Health Sciences, Division of Institute for Risk Assessment Sciences (IRAS), Pharmacy, \\ Utrecht University, 3584 CM, Utrecht, the Netherlands
}

\section{ABSTRACT}

There is increased concern about the selection pressure of antimicrobial use (AMU) in humans as well in farm animals resulting in antimicrobial (AM) resistance. The introduction of monitoring of AMU in foodproducing animals since 2011 has led to a considerable quantitative reduction of $\mathrm{AMU}$ in those animal species in the Netherlands. This survey was conducted to explore the possibilities to improve prudent use of AM in the cattle industry. We sent an online questionnaire to 373 veterinarians and asked which antimicrobial prophylaxis they used for their most recently performed cesarean section (CS) and left displaced abomasum (LDA) correction. With a response rate of $30 \%$, we found that older graduates used more AM for CS than recently graduated veterinarians (odds ratio $=2.4$ to 2.7 depending on category), whereas antimicrobial prophylaxis in LDA surgeries was significantly different for the available surgical correction methods. The results indicate that the respondents do not adjust the surgical antimicrobial prophylaxis for different conditions of the patient or the environment; $37(38 \%)$ respondents consider that the risks of contamination are similar for CS and LDA. In CS and all LDA correction methods preoperative prophylaxis was significantly less often applied than postoperative antimicrobial treatments (odds ratio $=0.05$ and 0.08 , respectively). When preoperative prophylaxis was applied, the choice of (combinations of) the AM and the timing of administration were not adequate at the start of the surgical procedure $(88 \%$ for CS and $90 \%$ for LDA). We conclude that considerable qualitative (timing, choice of antimicrobial, route of administration) and quantitative (limit AMU to indicated procedures) improvement on antimicrobial prophylaxis

Received September 10, 2020.

Accepted March 31, 2021

*Corresponding author: r.jorritsma@uu.nl for CS and LDA is possible by appreciation of the risks of the type of surgery (CS, LDA) and its conditions as well as by selecting classes of AM and timing and routes of administration that result in effective drug concentrations at the start of the procedure.

Key words: prudent antimicrobial use, cattle, cesarean section, correction of abomasum displacement

\section{INTRODUCTION}

There is increased concern that the selection of antimicrobial (AM)-resistant microorganisms seriously limits the future treatment options of humans and animals in need of AM treatment and hampers the development of modern medicine. The first step in refining antimicrobial use (AMU) in livestock was the ban on use of AM for growth promotion, implemented in Europe in 2006. In the Netherlands, a national policy on AMU reduction was introduced in 2009, which was characterized by the introduction of guidelines, AMU monitoring of all farms (since 2011), and a benchmarking system per animal species. This system with compulsory and voluntary actions resulted in an overall reduction in AMU of almost 70\% in 2019 compared with the situation in 2009 (Speksnijder et al., 2015; NethMap-MARAN, 2020).

The monitoring system showed that there were large differences in AMU between different sectors of the livestock industry. The Dutch dairy sector had the lowest AMU, which coincided with a limited AM resistance in dairy cows in comparison to other livestock animal species. Nevertheless, a further reduction in usage was achieved in dairy cattle. This was largely due to the introduction of selective dry cow treatment, as most AM treatments in dairy cattle were given intramammarily (SDa, 2014). An evaluation of this selective dry cow treatment strategy showed that this reduction neither resulted in an increased number of mastitis treatments, nor deteriorated udder health in general (Vanhoudt et al., 2018). 
In general, it is assumed that the risk of AM resistance selection is high for particular mass medication strategies, typically by oral administration and as part of standard practice, and this is the justification for monitoring and benchmarking the quantitative use of AM to achieve overall reduction of AMU.

Nevertheless, prudent AMU includes also the justified selection of the appropriate AM drug, including the dosage, timing, and duration of the treatment during therapeutic procedures such as surgery on individual animals. The efficacy of AM treatment depends on the proper AM substance for the (pathogenic) microbes on one hand, and the achieved serum and tissue levels of the drug on the other hand. These variables are determined by the drug formulation, dosing, route, and timing of administration and are described in several pharmacokinetic studies. For example, it is shown in cows that intramuscular administration of ampicillin sodium resulted in much faster and greater plasma concentrations when compared with 4 times higher dosages with intramuscular ampicillin anhydrate injections, although the duration of effective plasma concentrations was much shorter (Klein et al., 1989).

It is common practice to administer AM prophylaxis for surgical procedures in cows. The AM prophylaxis may be given before, during or after the surgical procedure, but there is strong evidence in humans that it is most effective when given before the procedure (Berríos-Torres et al., 2017). The objective of AM prophylaxis is not to sterilize tissues or to compensate for insufficient cleaning and disinfection of the surgical site, but to reduce the microbial burden of unavoidable intraoperative contamination. In human medicine, the recommended use of AM prophylaxis is based on a surgical wound classification such as the classification of the US National Research Council (Mangram et al., 1999). This classification is based on the estimated level of the bacterial contamination from the gastrointestinal tract and from the environment of the patient. The classification is also cited in the veterinary literature, but the compliance to the recommendations is unknown (Dumas et al., 2016).

To promote the prudent use of AM in the dairy industry, the objective of this study was to explore the use of AM prophylaxis for surgeries performed by Dutch veterinarians to identify areas for improvement. For this purpose, a national cross-sectional study was designed to describe and analyze the perioperative use of AM for 2 important surgical interventions in cows: cesareans and corrections of abomasum displacements to the left.

\section{MATERIALS AND METHODS}

\section{Survey}

We developed an online questionnaire on prophylactic use of AM for cesarean section (CS) and more or less invasive corrections of left displaced abomasum (LDA). In the Netherlands, these surgeries are always performed on the farm. The online questionnaire was tested in a small group of colleagues before sending it to the target population of all 373 veterinarians of the Royal Dutch Veterinary Association (KNMvD) with a membership in bovine medicine. In accordance with privacy legislation, the contact details of the veterinarians were not accessible to the authors, but were directly transferred by an independent operator into an online software system (EvaSys V7.0; https://evasys .de/en/). The self-administered questionnaire consisted of 14 open and 17 multiple-choice questions. To increase attention for the survey, the authors announced the study in their network using social media. The software system mailed nonresponders a reminder after 2 wk. To avoid a response bias, the veterinarians were able to submit their answers anonymously, although they could disclose their contact details at the end of the questionnaire to receive updates on the study. It was explicitly stated that all questions should be answered for the most recently performed LDA correction and CS to reduce recall bias. We used personalized links to minimize the risk that respondents submitted their answers more than once.

The questions were divided into 3 sections. The first section included questions about sex, working experience, working time spent with cattle, time since LDA correction and CS were last performed, and availability of specific protocols for the usage of AM around abdominal surgeries in their practice. Sections 2 and 3 of the survey included questions about the dosage, timing, application method, and AM used around CS and LDA correction, respectively. Veterinarians who performed a surgical LDA correction were asked to characterize the technique they used during the last procedure. In case of CS, we asked whether the veterinarian administered intrauterine AM during the last procedure. For both CS and LDA, we asked whether and, if applicable, which AM wound spray was used. Additionally, we asked the veterinarians to assess the cleanliness of the cow and the surgery site on a scale of 1 (very clean) to 5 (very dirty) of the most recent CS and LDA procedures. Finally, we asked the veterinarian to estimate the risk for complications after both surgeries with the used AM 
treatments and also for the (imaginary) situation where no AM were used. Respondents who did not perform a CS or an LDA correction were excluded from the data analyses.

\section{Data Handling}

Data were downloaded from the survey software into Microsoft Excel 2008 to calculate descriptive statistics, and we used R (version 4.0.2; https://www.r-project .org/) for statistical analyses. We used separate logistic models for LDA and CS to evaluate the effects of the following a priori set of categorical variables on AMU (yes/no): years of experience in clinical practice $(0-10$ yr, 10-20 yr, 20 to $30 \mathrm{yr}$, more than $30 \mathrm{yr}$ ), sex (male or female), percentage of available time dedicated to cattle (0-50\%, 50-75\%, more than 75\%), production type of the animal (meat or milk or dual purpose), cleanliness of the cow scored by the respondent (very clean, clean, average, dirty, or very dirty), cleanliness of the environment during the surgery scored by the respondent (very clean, clean, average, dirty, or very dirty), technique used (only for LDA), and number of cesareans performed by the respondent per month or the number of LDA surgeries per year $(0-2,2-4,4-6$, more than 6$)$. Odds ratios (OR) were derived from the estimators of the logistic model $\left(\mathrm{OR}=\mathrm{e}^{-\beta}\right)$. We analyzed timing and site of administration of the AM relative to the surgical procedure (pre-operative injection, intraabdominal use during the surgery, intraincisional between the muscle layers during the surgery, intramuscular injection after the surgery, spray on the closed wound, and for CS also the intrauterine application of AM), their interactions, as well as random effects for the respondents.

Two other logistic models were used to evaluate whether observations during the surgery affected the length of the postoperative AM treatment period as dependent variable (up to $3 \mathrm{~d}$ or longer). Included independent variables were the presence of asepsis failure during the surgery (yes/no), cleanliness of the animal, and cleanliness of the environment.

We used logistic regression to test whether the LDA correction method was associated with a higher or lower estimated risk of complications between CS and LDA. In addition, we used the exact binomial method to test whether the estimated risk on complications was higher for CS or LDA. Based on the estimations of the respondents of the risks for complications with the AM prophylaxis used and the estimated risk for complications without any AM prophylaxis, we calculated their estimated number needed to treat (NNT) for both surgeries as follows:

$$
\begin{aligned}
\mathrm{NNT}= & \frac{1}{\text { (risk for complications without prophylaxis })} . \\
& -(\text { risk for complications with prophylaxis })
\end{aligned}
$$

We used linear mixed effects models to compare the estimated NNT for CS and LDA, and we used Akaike information criterion for all model reductions.

\section{RESULTS}

\section{Survey Response Rate and Respondent Demographics}

A total of 113 out of 373 veterinarians completed the online survey, which corresponds to a response rate of $30 \%$. We found that $74 \%$ and $64 \%$ of the respondents had a specific protocol on AMU at their disposal for CS and LDA corrections, respectively. Other descriptive results are shown in Table 1, which are similar to the demographics of bovine veterinarians in the country (data not shown).

\section{Cesarean Sections}

A total of 111 of the 113 respondents performed a CS. All 111 respondents reported that they treated their last CS cow with one or more perioperative AM treatments. Almost all respondents (94\%) stated that they postoperatively administered AM intramuscularly.

Table 1. Characteristics of the respondents to a survey about antimicrobial use for cesarean section and left displaced abomasum performed by bovine veterinarians in the Netherlands

\begin{tabular}{lc}
\hline Item & $\mathrm{n}(\%)$ \\
\hline Sex & \\
Male & $89(78.8)$ \\
Female & $24(21.2)$ \\
Years of work experience & $21(18.6)$ \\
$0-10$ & $28(24.8)$ \\
$10-20$ & $35(31.0)$ \\
$20-30$ & $29(25.7)$ \\
$\geq 30$ & $2(1.8)$ \\
Percentage of time dedicated to cattle & $10(8.8)$ \\
$0-25^{1}$ & $24(21.2)$ \\
$25-50^{1}$ & $77(68.1)$ \\
$50-75$ & $9(8.0)$ \\
$75-100$ & \\
Number of veterinarians in practice & $26(23.0)$ \\
$1-3$ & $27(23.9)$ \\
$3-5$ & $51(45.1)$ \\
$5-8$ & \\
$\geq 8$ &
\end{tabular}

${ }^{1}$ Due to low numbers per class, these were combined into one during the statistical analyses. 
Even more respondents (98\%) sprayed the wound after closure, this spray contained AM in 30 cases. Many veterinarians (76/111) administered AM at more than one time point and $13(17 \%)$ of these 76 respondents used AM before, during, and after the CS. Fifty-two (47\%) of 111 respondents administered AM preoperatively and 44 (40\%) respondents administered AM intraoperatively.

Antimicrobial use for CS was not affected by the percentage of time dedicated to cattle, production type of the animal, cleanliness of the cow, cleanliness of the environment, number of performed CS per month, and sex of the respondent. Only timing, site of administration, and years of experience were significantly different in the logistic regression model. The use of AM spray on the closed wound was similar to the postoperative AMU, but pre-operative AMU $(\mathrm{OR}=0.05)$, intraabdominal AMU $(\mathrm{OR}=0.02)$, intraincisional AMU, between the muscle $\mathrm{AMU}(\mathrm{OR}=0.02)$, and intrauterine AMU $(\mathrm{OR}=0.06)$ were significantly $(P<0.05)$ less often practiced compared with postoperative AMU. Overall, respondents with 10 or more years of experience in practice used AM more often $(P<0.05)$ for CS compared with respondents with less experience $(\mathrm{OR}=$ 2.4 to 2.7 depending on category; Table 2).

Most respondents rated the cleanliness of the cow and the environment, respectively, as clean (score 2, $40 \%$ and $30 \%$ ) or normal (score 3, 35\% and $48 \%$ ). Many $(75 \%)$ stated that they finished the CS without intraoperative complications that resulted in unexpected infections or asepsis, whereas $13 \%$ encountered minor complications and only $12 \%$ encountered serious complications in asepsis. These factors and also sex were not associated with a postoperative AM treatment period of more than $3 \mathrm{~d}$.
From the 52 pre-operative treatments, 39 were given by intramuscular administration of ampicillin, procaine benzylpenicillin, or procaine benzylpenicillin-streptomycin combinations. One veterinarian administered the $\mathrm{AM}$ between 30 and $45 \mathrm{~min}$ before the incision, and 38 reported administering within 30 or 15 min before the first incision. The remaining 13 pre-operative treatments consisted of intravenously administered ampicillin sodium within 30 to 15 min before the first incision.

\section{Left Displaced Abomasum Corrections}

Table 3 shows which surgical techniques were used by the 110 respondents who performed a LDA correction and how often AM prophylaxis was used. Seventy-nine (72\%) of 110 respondents administered AM for their most recently performed LDA correction. Twenty-two of 110 (20\%) respondents administered AM preoperatively and $17(15 \%)$ of 110 respondents administered AM intraoperatively during LDA corrections by either intraabdominal AMU or intraincisional AMU between the muscle layers, and $72(65.5 \%)$ administered AM postoperatively. Even more respondents (92\%) sprayed the wound after closure; this spray contained AM in 37 cases.

Similar to AMU for CS, AMU for LDA was not affected by the percentage of time dedicated to cattle, production type of the animal, cleanliness of the cow, cleanliness of the environment, number of performed cesareans per year, and sex of the respondent. While most respondents indicated to administer postoperatively intramuscular AM, significantly less $(P<0.05)$ AM were administered pre-operatively $(\mathrm{OR}=0.08)$, in the abdomen $(\mathrm{OR}=0.03)$, or intraincisionally between the muscle layers $(\mathrm{OR}=0.03)$. In contrast with

Table 2. Reported antimicrobial use (\% in parentheses) in cesarean section and corrections of left displaced abomasum (LDA) for respondents with different years of work experience

\begin{tabular}{|c|c|c|c|}
\hline \multirow[b]{2}{*}{ Item } & \multicolumn{3}{|c|}{$\begin{array}{l}\text { Antimicrobial administration at different } \\
\text { surgical periods }\end{array}$} \\
\hline & Pre & Intra & Post \\
\hline \multicolumn{4}{|l|}{ Cesarean section } \\
\hline \multicolumn{4}{|l|}{ Years of work experience } \\
\hline $0-10(\mathrm{n}=20)$ & $7(35)$ & $2(10)$ & $19(95)$ \\
\hline $10-20(\mathrm{n}=27)$ & $12(44)$ & $13(48)$ & $27(100)$ \\
\hline $20-30(\mathrm{n}=35)$ & $21(60)$ & $15(43)$ & $31(89)$ \\
\hline$>30(\mathrm{n}=29)$ & $12(41)$ & $14(48)$ & $27(93)$ \\
\hline Total antimicrobial use $(\mathrm{n}=111)$ & $52(47)$ & $44(40)$ & $104(94)$ \\
\hline \multicolumn{4}{|l|}{ LDA correction } \\
\hline \multicolumn{4}{|l|}{ Years of work experience } \\
\hline $0-10(\mathrm{n}=20)$ & $5(25)$ & $1(5)$ & $14(70)$ \\
\hline $10-20(\mathrm{n}=27)$ & $6(22)$ & $3(11)$ & $15(56)$ \\
\hline $20-30(\mathrm{n}=35)$ & $7(20)$ & $4(11)$ & $22(63)$ \\
\hline$>30(\mathrm{n}=28)$ & $4(14)$ & $9(32)$ & $21(75)$ \\
\hline Total antimicrobial use $(\mathrm{n}=110)$ & $22(20)$ & $17(15)$ & $72(65)$ \\
\hline
\end{tabular}


CS, AMU for LDA was not associated with years of experience (Table 2). Compared with the laparoscopic technique, AMU was significantly $(P<0.05)$ higher when respondents used left flank laparotomy $(\mathrm{OR}=$ 7.3), right flank laparotomy $(\mathrm{OR}=6.1)$, and less when other techniques were used $(\mathrm{OR}=0.26)$.

Fourteen of the 22 pre-operative treatments consisted of intramuscular administering of ampicillin or procaine benzylpenicillin given within 30 to 15 min before the first incision. The remaining 8 pre-operative treatments consisted of intravenously administered ampicillin sodium within 30 or 15 min before the first incision.

Postoperative AMU was reported by $72(65 \%)$ respondents, although $85 \%$ of the LDA corrections were performed without intraoperative complications, $11 \%$ with minor complications, and only $4 \%$ with serious complications in asepsis. Most respondents rated the cleanliness of the cow and the environment, respectively, as clean (score 2, $57 \%$ and $42 \%$ ) or normal (score 3, 32\% and 47\%). Postoperative AM treatments were almost always given for 1 to $3 \mathrm{~d}$ (94.5\%). Similar to CS, we found no associations between the cleanliness of the cow, cleanliness of the environment, occurrence of complications, or sex and the length of the postoperative AM treatment period.

\section{Number Needed to Treat}

We found that 37 of the respondents indicated that the perceived risk of complications due to contaminations during the surgery was similar between CS and LDA. There was no effect of the applied LDA correction technique in the analyses, which means that the perceived risks for these techniques were similar. The respondents indicating that the perceived risk between CS and LDA was not similar, estimated in a significant majority ( $88 \%$, CI $77-95 \%)$ that the risk of complications was higher for CS compared with LDA. After deriving the NNT for both CS and LDA based on the perceived risk of the veterinarians for complications with and without AM prophylaxis, we calculated that this derived NNT was significantly $(P<0.05)$ lower for CS (5.6) compared with LDA (7.9). This means that in case of LDA, more treatments are thought to be necessary to prevent one cow with complications compared with CS. The point estimate of the effect was $2.32(95 \%$ $\mathrm{CI}=1.24-3.40)$.

\section{Product Selection and Timing of Antimicrobial Prophylaxis}

Figure 1 illustrates which AM were administered as pre-operative, intraoperative, and postoperative surgical prophylaxis. Overall, procaine benzylpenicillin, aminopenicillins, and combinations of these AM with aminoglycosides were most frequently used. Other administered AM included gentamicin and oxytetracycline.

\section{DISCUSSION}

The results of this survey suggests that the estimated risks on complications are not always consistent with general principles and with AMU. Many respondents stated that these risks were similar between CS and LDA, similar for different environmental conditions, and similar between the LDA correction methods, but the AMU was in many cases not the same. We also found that postoperative treatments were always part of AM prophylactic use and often combined with many other treatments. It appeared that the respondents use many different AM products.

By approaching all veterinarians with a special membership on bovine medicine and obtaining a fair response rate of $30 \%$, we think that the answers are representative for bovine veterinarians in the Netherlands. We do realize that limiting bias continues to be a challenge in many surveys. To address this, we specifically asked what they did during their most recent CS and LDA correction to limit recall bias. We think that this bias is in particular present when a respondent does not perform these interventions very frequently, which is to our knowledge in general not the case for bovine veterinarians in the Netherlands. It is possible that the most recently performed surgery is not representative for most of the interventions, but we think

Table 3. Antimicrobial use (\% in parentheses) for different techniques for the correction of left displaced abomasum

\begin{tabular}{|c|c|c|}
\hline Technique & Used antimicrobials & Used no antimicrobials \\
\hline Laparoscopic abomasopexy $(\mathrm{n}=26)$ & $11(42)$ & $15(58)$ \\
\hline Left flank laparotomy $(\mathrm{n}=26)$ & $23(88)$ & $3(12)$ \\
\hline Right flank laparotomy $(\mathrm{n}=45)$ & $42(93)$ & $3(7)$ \\
\hline Other techniques $^{1}(\mathrm{n}=15)$ & $3(20)$ & $12(80)$ \\
\hline Overall & $79(72)$ & $31(28)$ \\
\hline
\end{tabular}

${ }^{1}$ This includes roll and toggle, roll and suture, laparotomy on a lying cow, and laparoscopy on a lying cow. 


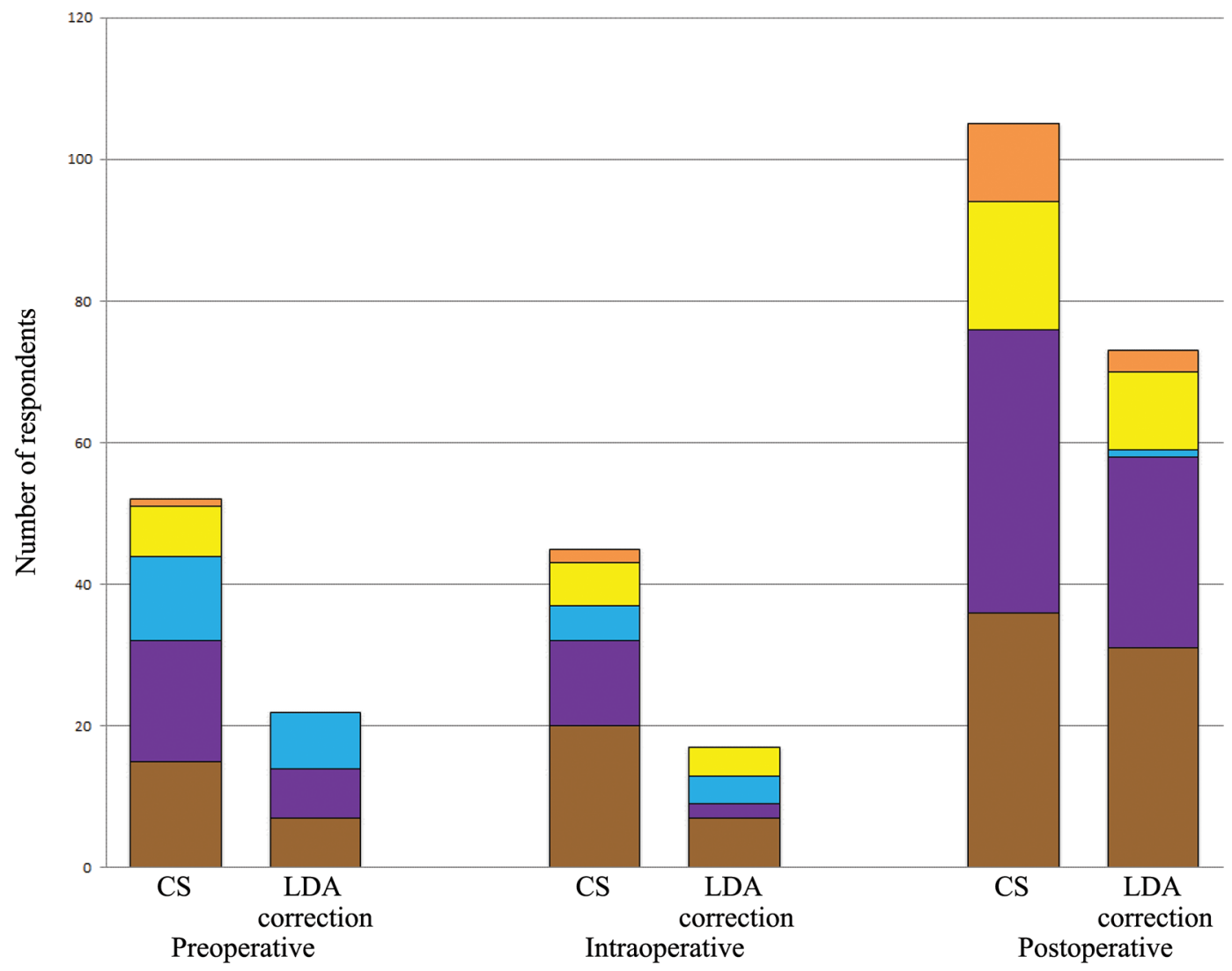

Figure 1. Frequency of perioperative antibiotic use for cesarean section (CS, n = 111) and surgical left displaced abomasum (LDA) corrections $(\mathrm{n}=110)$ by Dutch practitioners. Brown $=$ procaine benzylpenicillin; purple $=$ ampicillin trihydrate; blue $=$ ampicillin sodium; yellow $=$ procaine benzylpenicillin-streptomycin combinations; and orange $=$ others.

that this is limited by the relatively high availability of protocols for surgical prophylaxis. We intended to minimize response bias with the possibility to submit answers anonymously.

The need for surgical prophylaxis in human medicine is confined by protocols for the adequate selection of treatment regimens. It was previously indicated that the availability and compliance to protocols in veterinary medicine is probably rather low (Hardefeldt et al., 2017a,b). The Dutch policy to reduce the number of AM treatments in animals may have increased the awareness of prudent AMU and might have attributed to the relatively high availability of protocols for the surgical prophylaxis of CS (74\%) and LDA (64\%) in our study.

We found a high frequency of prophylactic AMU for both CS and LDA. In CSs, $100 \%$ of the respondents reported the use of prophylactic AM and $72 \%$ of the respondents reported prophylactic AMU for LDA corrections. Similar studies in Canada, Australia and Belgium on the use of prophylactic AM in bovine abdominal surgeries reported comparable results (Chicoine et al., 2008; Hardefeldt et al., 2017a; Djebala et al.,
2019). While it could be speculated that veterinarians who graduated up for example 5 yr ago use more AM to compensate for being less skilled or less quickly in performing the procedure, we found that the opposite was true for respondents doing CS as more recently started veterinarians used significantly less AM. We speculate that the increased attention for prudent AMU in veterinary education influenced the behavior of recent graduates. Another explanation is that the class with 0 to $10 \mathrm{yr}$ of experience also includes the very recently graduated veterinarians, showing less risk aversive behavior due to the fact that they have not seen many cows with severe complications yet.

We did not observe a similar effect of years in practice on AMU for LDA, but we found significantly more AM treatments for left and right flank laparotomies compared with other techniques, such as laparoscopic corrections and the roll and toggle method. The differentiation in prophylaxis suggests that veterinarians adapt their AM strategy to the circumstances, but they do not closely follow the human wound classification system. It appears that AM prophylaxis is found more important for the more invasive than for the less inva- 
sive techniques, such as laparoscopic corrections and the roll and toggle method. This may suggest that veterinarians are concerned about creating and maintaining aseptic conditions for surgeries at the farm, because the less invasive techniques are characterized by a higher bacterial contamination from the gastro-intestinal tract. An observational study indicates that right flank pyloric omentopexy of prolonged duration in an educational environment requires more often postoperative antibiotic treatments than a 2-step laparoscopy guided abomasopexy (Roy et al., 2008). Whether in another setting laparoscopic abomasopexy or roll and toggle are procedures associated with higher or lower postoperative infection risks than right flank pyloric omentopexy, and therefore prone to more or less AM prophylaxis, is an issue which is not fully addressed up till now.

Another explanation for the observed differences between the LDA techniques could be that the estimated physical condition of the cows selected for laparotomy was worse compared with those that underwent laparoscopy or roll and toggle, which urged the veterinarians more often to use AM prophylaxis.

The result of this questionnaire indicates that the actual conditions before and during the surgical procedure, such as the cleanliness of the environment, the cleanliness of the animal and the occurrence of complications do not significantly influence the regimen of AM prophylaxis selected by the respondents. It is possible that the perceived risks on complications due to these conditions are quite similar and do not require any adjustment of the prophylaxis. On the other hand, it is also possible that the veterinarians are reluctant to take these issues into account as they are risk aversive or not able to quantify the impact on the risk of surgical site infections and just use the prophylaxis that is perceived effective under all circumstances. Increased attention and quantification of the risks may in that case result in tailored and thus a reduced use of AM, but we are not aware of the presence of quantitative tools to support the veterinarian in this task.

Although the majority of the respondents indicated that the risks of complications due to contaminations during the procedure were higher for CS than for LDA, we found that 37 respondents estimated that these risks were similar. As we found no effects of the number of LDA or CS interventions per year or per month, is it not likely that this difference in estimated risk is explained by experience. Also, the results indicate that the estimated risks of complications were found to be similar for the different LDA techniques, which contrast with the differentiated AM strategy for these techniques. Most LDA procedures should be classified as clean, whereas the contamination in others is very limited compared with the CS procedures, which are in general classified as clean-contaminated according to the human wound classification system. The reason for classifying CS procedures as clean-contaminated is that it is not possible to prevent at least some leakage of fetal fluids into the abdominal cavity. The validity of this classification is supported by our study on the risks of complications between CS and explorative laparotomy in cows (Jorritsma et al., 2018). Consequently, the risks of surgical complications for LDA are lower than for CS as it seems unlikely that in clinical practice the encountered clinical condition of the patients or the environmental conditions are worse for LDA compared with CS. Therefore, we think that there is room for improvement in surgical site infection prophylaxis in dairy cattle. Recognition of the necessary differentiation in AM prophylaxis between LDA and CS by practitioners should be attained, and the elsewhere reported NNT of 53 for LDA to prevent complication substantiates that it is possible to perform many LDA surgeries without any AM prophylaxis (Jorritsma et al., 2018). The question is at what (objective) NNT prophylactic treatment is justified, but both the NNT of 53 for LDA and the NNT of 29 for CS to prevent complications are substantially higher than the perceived NNT of the practitioners (Jorritsma et al., 2018). This means that the veterinarians seem to overestimate the need for or effect of AM prophylaxis.

While optimal surgical prophylaxis is achieved when effective concentrations of AM are present from the start of the procedure, the respondents in our study applied significantly more often the less effective postoperative administration of AM (Haven et al., 1992; de Jonge et al., 2017). For optimal prophylaxis, it is necessary to select AM with the appropriate spectrum in combination with suitable pharmacodynamic and pharmacokinetic characteristics (Boothe and Boothe, 2015). In case of CS, surgical contamination from the uterus contains a mixed population of gram-negative and gram-positive bacteria (Mijten et al., 1997; Mao et al., 2015), whereas gram-negative bacteria such as Prevotella spp., and Butyrivibrio spp. are primarily isolated from the abomasum. In general, gram-positive bacteria such as Streptococcus spp., Staphylococcus spp., and Bacillus spp. are frequently isolated from the skin. Other bacteria such as Trueperella pyogenes, Escherichia coli, and Fusobacterium necrophorum were cultured from peritoneal and incisional infections (Hoeben et al., 1997). It seems therefore appropriate that many respondents selected broad-spectrum AM for surgical prophylaxis, although procaine benzylpenicillin accounted for 20 to $50 \%$ of the chosen AM prophylaxis.

Intravenous administration of ampicillin sodium solution will reach maximum plasma concentration within minutes after injection, whereas this will take around 
15 to 20 min after intramuscular administration of this solution (Klein et al., 1989; Fernández-Varón et al., 2005). Even when dosed 4 times higher, intramuscular injections of procaine benzylpenicillin and ampicillin trihydrate achieved their lower maximum plasma concentration only after at least $3 \mathrm{~h}$ (Dubreuil et al., 2001; Credille et al., 2015).

Comparable information is available in other papers for benzylpenicillin in sodium or potassium salt, or as a procaine ester. Bengtsson et al. (1989) showed that administration of the procaine ester in 4 times higher doses than intramuscular administration of the potassium salt results in $30 \%$ of the serum levels, whereas another study demonstrated that the intramuscular administration of the potassium salt results in a maximum level of only $30 \%$ in comparison with the same dose administered intravenously as the sodium salt (Hekman et al., 1982). These differences between chemical formulations and routes of administration were nicely illustrated in pharmacokinetic modeling by Li et al. (2014).

We therefore estimate that effective prophylaxis was only present for the 13 of the 111 CS (12\%) and the 8 of the $79(10 \%)$ LDA cases that used ampicillin sodium. The other strategies likely failed to reach effective concentrations at the start of the surgery and should be regarded as inferior.

Many respondents stated that they used intraabdominal or intraincisional administration of AM. We agree with Lyons et al. (2013) that there is no evidence that these routes of administration have any additional effect on top of pre-operative prophylaxis. It is demonstrated that that intraabdominal administration of AM may cause inflammatory reactions (Klein et al., 1989). Also, the intraabdominal or intraincisional administration routes are usually not approved, should therefore require adjusted withdrawal times in many countries, and are therefore severely criticized (Djebala et al., 2019). Approximately one-third of the respondents used a spray with AM to cover the wound. While we are not aware of clinical studies on cows, human studies suggest that topical AM change the ecological microbiota balance at the surgical site and might have more negative consequences for the patient than wounds that are not treated with AM (Romano-Bertrand et al., 2015).

\section{CONCLUSIONS}

We conclude that improvement of AM prophylaxis for CS and LDA surgeries is possible by appreciation of the risks of the type of surgery and its conditions in around $90 \%$ of the procedures by selecting AM and routes of administration that are effective at the start of the surgery. As the quantitative AMU and thus the effect on the selection of AM resistance in dairy is prob- ably quite low, we think that this improvement would be the next step toward the prudent use of antibiotics in the dairy industry. We found it encouraging that recently graduated respondents were more restrictive in the use of AM.

\section{ACKNOWLEDGMENTS}

We thank Jan van den Broek (Utrecht University) for the statistical analysis of the data and the many veterinarians who completed the questionnaire. We are grateful for the help of the Royal Dutch Veterinary Association (KNMvD; Houten, the Netherlands) for their collaboration in the distribution of the questionnaire. We declare no conflict of interest. We received no external funding for this study.

\section{REFERENCES}

Bengtsson, B., A. Franklin, J. Luthman, and S. O. Jacobsson. 1989. Concentrations of sulphadimidine, oxytetracycline and penicillin $\mathrm{G}$ in serum, synovial fluid and tissue cage fluid after parenteral administration to calves. J. Vet. Pharmacol. Ther. 12:37-45. https: //doi.org/10.1111/j.1365-2885.1989.tb00639.x.

Berríos-Torres, S. I., C. A. Umscheid, D. W. Bratzler, B. Leas, E. C. Stone, R. R. Kelz, C. E. Reinke, S. Morgan, J. S. Solomkin, J. E. Mazuski, E. Patchen Dellinger, K. M. F. Itani, E. F. Berbari, J. Segreti, J. Parvizi, J. Blanchard, G. Allen, J. A. J. W. Kluytmans, R. Donlan, W. P. Schecter, and Healthcare Infection Control Practices Advisory Committee. 2017. Centers for Disease Control and Prevention Guideline for the prevention of surgical site infection. J. Am. Med. Assoc. Surg. 152:784-791. https://doi.org/10.1001/ jamasurg.2017.0904.

Boothe, D. M., and H. W. Boothe. 2015. Antimicrobial considerations in the perioperative patient. Vet. Clin. North Am. Small Anim. Pract. 45:585-608. https://doi.org/10.1016/j.cvsm.2015.01.006.

Chicoine, A. L., P. M. Dowling, J. O. Boison, and S. Parker. 2008. A survey of antimicrobial use during bovine abdominal surgery by western Canadian veterinarians. Can. Vet. J. 49:1105-1109.

Credille, B. C., S. Giguère, T. W. Vickroy, H. J. Fishman, A. L. Jones, M. E. Mason, R. O. DiPietro, and D. T. Ensley. 2015. Disposition of ampicillin trihydrate in plasma, uterine tissue, lochial fluid, and milk of postpartum dairy cattle. J. Vet. Pharmacol. Ther. 38:330-335. https://doi.org/10.1111/jvp.12178.

de Jonge, S. W., S. L. Gans, J. J. Atema, J. S. Solomkin, P. E. Dellinger, and M. A. Boermeester. 2017. Timing of preoperative antibiotic prophylaxis in 54,552 patients and the risk of surgical site infection: A systematic review and meta-analysis. Medicine (Baltimore) 96:e6903. https://doi.org/10.1097/MD.0000000000006903.

Djebala, S., M. Moula, C. Bayrou, A. Sartelet, and P. Bossaert. 2019. Prophylactic antibiotic usage by Belgian veterinarians during elective caesarean section in Belgian blue cattle. Prev. Vet. Med. 172:104785. https://doi.org/10.1016/j.prevetmed.2019.104785.

Dubreuil, P., J. Daigneault, Y. Couture, P. Guay, and D. Landry. 2001. Penicillin concentrations in serum, milk, and urine following intramuscular and subcutaneous administration of increasing doses of procaine penicillin $\mathrm{G}$ in lactating dairy cows. Can. J. Vet. Res. 65:173-180.

Dumas, S. E., H. M. French, S. N. Lavergne, C. R. Ramirez, L. J. Brown, C. R. Bromfield, E. F. Garrett, D. D. French, and B. M. Aldridge. 2016. Judicious use of prophylactic antimicrobials to reduce abdominal surgical site infections in periparturient cows: Part 1 - A risk factor review. Vet. Rec. 178:654-660. https://doi.org/ $10.1136 / v r . i 103677$. 
Fernández-Varón, E., E. Escudero-Pastor, and C. M. Cárceles-Rodríguez. 2005. Pharmacokinetics of an ampicillin-sulbactam combination after intravenous and intramuscular administration to neonatal calves. Vet. J. 169:437-443. https://doi.org/10.1016/j.tvjl .2004.03.001.

Hardefeldt, L. Y., G. F. Browning, K. A. Thursky, J. R. Gilkerson, H. Billman-Jacobe, M. A. Stevenson, and K. E. Bailey. 2017a. Crosssectional study of antimicrobials used for surgical prophylaxis by bovine veterinary practitioners in Australia. Vet. Rec. 181:426. https://doi.org/10.1136/vr.104375.

Hardefeldt, L. Y., G. F. Browning, K. Thursky, J. R. Gilkerson, H. Billman-Jacobe, M. A. Stevenson, and K. E. Bailey. 2017b. Antimicrobials used for surgical prophylaxis by companion animal veterinarians in Australia. Vet. Microbiol. 203:301-307. https:// doi.org/10.1016/j.vetmic.2017.03.027.

Haven, M. L., J. J. Wichtel, D. G. Bristol, J. F. Fetrow, and J. W. Spears. 1992. Effects of antibiotic prophylaxis on postoperative complications after rumenotomy in cattle. J. Am. Vet. Med. Assoc. 200:1332-1335.

Hekman, P., J. F. Nouws, and C. A. van Ginneken. 1982. Effect of Tomanol on the pharmacokinetics and tissue distribution of penicillin G in dairy cows. Vet. Q. 4:12-18. https://doi.org/10.1080/ 01652176.1982 .9693832$.

Hoeben, D., P. Mijten, and A. de Kruif. 1997. Factors influencing complications during caesarean section on the standing cow. Vet. Q. 19:88-92. https://doi.org/10.1080/01652176.1997.9694748.

Jorritsma, R., I. M. van Geijlswijk, and M. Nielen. 2018. Randomized prospective trials to study effects of reduced antibiotic usage in abdominal surgery in cows. J. Dairy Sci. 101:8217-8223. https:// doi.org/10.3168/jds.2017-14158.

Klein, W. R., E. C. Firth, J. M. Kievits, and J. C. de Jager. 1989. Intra-abdominal versus intramuscular application of two ampicillin preparations in cows. J. Vet. Pharmacol. Ther. 12:141-146. https:/ /doi.org/10.1111/j.1365-2885.1989.tb00655.x.

Li, M., R. Gehring, L. Tell, R. Baynes, Q. Huang, and J. E. Riviere. 2014. Interspecies mixed-effect pharmacokinetic modeling of penicillin G in cattle and swine. Antimicrob. Agents Chemother. 58:4495-4503. https://doi.org/10.1128/AAC.02806-14.

Lyons, N. A., S. Karvountzis, and T. J. D. Knight-Jones. 2013. Aspects of bovine caesarean section associated with calf mortality, dam survival and subsequent fertility. Vet. J. 197:342-350. https:/ /doi.org/10.1016/j.tvjl.2013.01.010.
Mangram, A. J., T. C. Horan, M. L. Pearson, L. C. Silver, and W. R. Jarvis. 1999. Guideline for Prevention of Surgical Site Infection, 1999. Am. J. Infect. Control. Outcomes Manag. 27:97-134.

Mao, S., M. Zhang, J. Liu, and W. Zhu. 2015. Characterising the bacterial microbiota across the gastrointestinal tracts of dairy cattle: Membership and potential function. Sci. Rep. 5:16116. https://doi .org/10.1038/srep16116.

Mijten, P., A. E. van den Bogaard, M. J. Hazen, and A. de Kruif. 1997. Bacterial contamination of fetal fluids at the time of cesarean section in the cow. Theriogenology 48:513-521. https://doi.org/10 1016/S0093-691X(97)00259-8.

NethMap-MARAN. 2020. Consumption of antimicrobial agents and antimicrobial resistance among medically important bacteria in the Netherlands - Monitoring of antimicrobial resistance and antibiotic usage in animals in the Netherlands in 2019. https://swab .nl/en/nethmap-pvid369.

Romano-Bertrand, S., P. Licznar-Fajardo, S. Parer, and E. JumasBilak. 2015. Environmental impact on human microbiota: Focus on hospitalization effects on skin and surgical microbiota. Rev. Francoph. Lab. 2015:75-82. https://doi.org/10.1016/S1773 $-035 \mathrm{X}(15) 72824-8$.

Roy, J. P., D. Harvey, A. Bélanger, and S. Buczinski. 2008. Comparison of 2-step laparoscopy-guided abomasopexy versus omentopexy via right flank laparotomy for the treatment of dairy cows with left displacement of the abomasum in on-farm settings. J. Am. Vet. Med. Assoc. 232:1700-1706. https://doi.org/10.2460/javma 232.11.1700.

SDa. 2014. Usage of Antibiotics in Agricultural Livestock in the Netherlands in 2014. Accessed Apr. 26, 2021. https://cdn.i-pulse .nl/autoriteitdiergeneesmiddelen/userfiles/Publicaties/def-sda -rapport-ab-2014-engels-v2-aangepast-102015-incl-erratum-(1) .pdf.

Speksnijder, D. C., D. J. Mevius, C. J. M. Bruschke, and J. A. Wagenaar. 2015. Reduction of veterinary antimicrobial use in the Netherlands. The Dutch success model. Zoonoses Public Health 62(Suppl 1):79-87. https://doi.org/10.1111/zph.12167.

Vanhoudt, A., K. van Hees-Huijps, A. T. M. van Knegsel, O. C. Sampimon, J. C. M. Vernooij, M. Nielen, and T. van Werven. 2018. Effects of reduced intramammary antimicrobial use during the dry period on udder health in Dutch dairy herds. J. Dairy Sci. 101:3248-3260. https://doi.org/10.3168/jds.2017-13555. 